\title{
Protective effects of resveratrol against 5-fluorouracil-induced oxidative stress and inflammatory responses in human keratinocytes
}

\author{
Shu Chen, ${ }^{1}$ Naofumi Tamaki, ${ }^{1, *}$ Yasusei Kudo, ${ }^{2}$ Takaaki Tsunematsu, ${ }^{3}$ Kaname Miki, ${ }^{1}$ Naozumi Ishimaru, ${ }^{3}$ and \\ Hiro-O Ito ${ }^{1}$
}

${ }^{1}$ Department of Preventive Dentistry, ${ }^{2}$ Department of Oral Bioscience, and ${ }^{3}$ Department of Oral Molecular Pathology, Tokushima University Graduate School of Biomedical Sciences, 3-18-15 Kuramoto-cho, Tokushima 770-8504, Japan

(Received 19 February, 2021; Accepted 11 June, 2021)

\begin{abstract}
Although 5-fluorouracil (5-FU) is currently used as an anti-cancer chemotherapy, adverse effects such as oral mucositis potentially limit its clinical application. Additionally, the prevention of 5-FUinduced side effects are scarce. Resveratrol is known to decrease oxidative damage and inflammation. In this study, we examined the protective effects of resveratrol on 5-FU-induced oxidative stress and inflammatory responses in normal human keratinocytes ( $\mathrm{HaCaT}$ cell) as in vitro oral mucositis model. $\mathrm{HaCaT}$ cells were exposed to 5-FU and simultaneously treated with resveratrol. The effects of resveratrol on 5-FU-induced cytotoxicity were evaluated using cell viability assay. The production of reactive oxygen species (ROS) was measured using a fluorescence spectrophotometer. The effects of resveratrol on nuclear factor erythroid 2-related factor 2 (Nrf2), silent information regulator transcript-1 (SIRT-1), and nuclear factor kappa B (NF-кB) signaling and inflammatory cytokine expression were examined. Resveratrol suppressed 5-FU-induced overproduction of ROS by upregulating anti-oxidant defense genes through Nrf2 activation and SIRT-1 expression. Concerning inflammatory responses, resveratrol suppressed the 5-FU-induced expression of proinflammatory cytokines via NF- $\mathrm{KB}$ nuclear translocation. Conversely, $\mathrm{N}$-acetylcysteine reduced ROS levels without affecting the expression of pro-inflammatory cytokines. Resveratrol might be useful for preventing 5-FU-induced adverse effects by activating anti-oxidant and anti-inflammatory responses.
\end{abstract}

Key Words: oxidative stress, inflammation, oral mucositis, resveratrol, human normal keratinocyte

$\mathrm{T}$ he pyrimidine anti-metabolite 5-fluorouracil (5-FU) is widely used to treat a variety of cancers; however, it causes several side effects. Oral mucositis, a painful inflammatory ulcer of the oral mucosa, is a common side effect occurring in up to $90 \%$ in patients with cancer treated with 5 -FU..$^{(1,2)}$ The clinical manifestation of oral mucositis is ulceration of the mucosal epithelium. In addition, the formation of ulcers causes severe pain, altered taste, dry mouth, and decreased appetite, and it directly or indirectly induces the suppression of nutritional uptake in many cases. ${ }^{(3-5)}$ Despite the availability of palliative treatments including antibiotics, analgesics, and radioprotective agents, in addition to general oral hygiene modification, these symptomatic therapies have limited efficacy. ${ }^{(6)}$

Recent studies illustrated that the pathogenesis of oral mucositis is based on reactive oxygen species (ROS) generation and inflammation. ${ }^{(2,3)}$ The overproduction of ROS leads to their accumulation and oxidative stress, which further aggravate cell damage and eventually cause apoptosis. ${ }^{(7)}$ Therefore, cells scavenge the overproduced ROS through anti-oxidant networks, and the balance between ROS production and scavenging is tightly regulated. ${ }^{(8)}$ Nuclear factor erythroid 2-related factor 2 (Nrf2) is a pleiotropic protein and important anti-oxidant sensor. Under oxidative stress, electrophiles and oxidants modify Kelchlike ECH-associated protein-1, leading to its dissociation from Nrf2. Following its release, Nrf2 translocates to the nucleus and then binds to an anti-oxidant response element (ARE) sequence to mediate the transcription of target genes, such as heme oxygenase $1(\mathrm{HO}-1)$ and NAD(P)H-quinone oxidoreductase (NQO-1). ${ }^{(9)}$ Transcription of these genes increases the resistance of cells to oxidative stress and protects against inflammation. ${ }^{(10)}$

Resveratrol is a polyphenolic phytoalexin that naturally occurs in many plant products, such as grapes, berries, and peanuts. ${ }^{(1)}$ It has been reported that resveratrol is more effective than conventional anti-oxidants such as $N$-acetylcysteine (NAC) and quercetin in decreasing oxidative damage. ${ }^{(12)}$ The cellular effects of resveratrol were attributed to its anti-inflammatory and antioxidant capacities. However, the protective effects of resveratrol on 5-FU-induced damage in normal keratinocytes remain to be determined. Furthermore, resveratrol has been reported to enhance 5-FU-induced cytotoxicity in cancer cells. ${ }^{(13)}$

In this study, a normal human keratinocyte cell line ( $\mathrm{HaCaT})$ was used as an in vitro model to investigate oral mucositis caused by 5-FU, as these cells can serve as a useful model for oral mucositis research. ${ }^{(14)}$ Several studies suggested that HaCaT cells can produce pro-inflammatory cytokines, such as interleukin (IL)-1, IL-6, ${ }^{(15)}$ and tumor necrosis factor (TNF)- $\alpha .^{(16)}$ However, the roles of ROS production and upregulation of the nuclear factor kappa $\mathrm{B}(\mathrm{NF}-\mathrm{kB})$ pathway induced by $5-\mathrm{FU}$ in normal keratinocytes have not been explored.

We hypothesized that resveratrol can prevent or attenuate oral mucositis caused by 5-FU. We evaluated the inhibitory effects of resveratrol on 5-FU-induced oxidative stress and inflammatory responses. Moreover, we investigated the effects of resveratrol on the Nrf2, silent information regulator transcript-1 (SIRT-1), and $\mathrm{NF}-\kappa \mathrm{B}$ signaling pathways.

\section{Materials and Methods}

Cell culture. The HaCaT immortalized keratinocyte cell line from adult human skin was obtained from German Cancer Research Center (Heidelberg, Germany). Cells were plated in cell culture flasks and suspended in Dulbecco's Modified Eagel

*To whom correspondence should be addressed.

E-mail: tamanaof@tokushima-u.ac.jp 
Table 1. Primers used in this study for real-time PCR

\begin{tabular}{lcc}
\hline Gene Name & \multicolumn{1}{c}{ Forward Primer } & Reverse Primer \\
\hline IL-1 $\beta$ & TGATGGCTTATTACAGTGGC & TGTAGTGGTGGTCGGAGATT \\
IL-6 & AAGCCAGAGCTGTGCAGATGAGTA & TGTCCTGCAGCCACTGGTTC \\
TNF- $\alpha$ & CCTCTGGCCAGGCAGTCAGA & GGCGTTTGGGAAGGTTGGAT \\
IL-10 & GCTGCACCCACTTCCCAGGC & CCTGCTCCACGGCCTTGCTC \\
HO-1 & CTTTCAGAAGGGTCAGGTGTC & TGCTTCTTTCGCTCTATCTCC \\
NQO-1 & CATCATTTGGGTCAAGTCC & ACAGCCGTGGCAGAACTA \\
SIRT-1 & AAGGAAAACTACTTCGCAAC & GGAACCATGACACTGAATTA \\
GAPDH & GTATTGGGCGCCTGGTCACC & CGCTCCTGGAAGAGATGGTGATGG \\
\hline
\end{tabular}

Medium (DMEM; Nacalai Tesque, Inc., Kyoto, Japan) supplemented with $10 \%$ fetal bovine serum (MP Biomedicals LLC, Santa Ana, CA), $100 \mathrm{U} / \mathrm{ml}$ penicillin, $0.1 \mathrm{mg} / \mathrm{ml}$ streptomycin (Sigma-Aldrich, St. Louis, MO), and kept in a humidified incubator at $37^{\circ} \mathrm{C}$, with an atmosphere of $5 \% \mathrm{CO}_{2}$.

Cell viability. The cell viability assay was conducted to determine the concentrations of resveratrol as a chemopreventive agent against toxicity induced by 5-FU. Briefly, HaCaT cells were seeded into the 96-well plate and incubated for $24 \mathrm{~h}$. Then cells treated with or without different concentrations of resveratrol and 5-FU for $24 \mathrm{~h}$. WST-1 (Takara Bio Inc., Otsu, Japan) assay was performed according to the standard protocol of manufacture. Absorbance value was measured at $450 \mathrm{~nm}$ by a microplate reader. Cell viability was expressed as the percentage of untreated cells (control).

Production and detection of ROS. HaCaT cells were incubated with 5-FU in presence or absence of resveratrol for 24 and $48 \mathrm{~h}$. The adherent cells were stained with CellROX Green Reagent (Life Technology, Carlsbad, CA) at $37^{\circ} \mathrm{C}$ in dark for $30 \mathrm{~min}$, nuclei were stained by 4',6-diamidino-2-phenylindole (DAPI) for $15 \mathrm{~min}$, and harvested in PBS. The cell suspension $(10,000$ cells/well) was placed in a flat bottom 96-well assay plate (Nunc/ThermoFisher scientific, Waltham, MA). The intensity was then immediately measured using the fluorescence spectrophotometer (Tecan M200 Infinite PRO, Tecan Japan Co, Kawasaki, Japan) at an excitation wavelength $485 \mathrm{~nm}$ and emission wavelength $520 \mathrm{~nm}$.

Immunofluorescence staining. $\mathrm{HaCaT}$ cells were incubated and then treated with 5-FU in the presence or absence of resveratrol for $24 \mathrm{~h}$. Subsequently, the cells were fixed with $4 \%$ paraformaldehyde for $15 \mathrm{~min}$ followed by permeabilized with $0.5 \%$ Triton X-100. The cells were blocked with Blocking One Histo (Nacalai Tesque) for $10 \mathrm{~min}$ to prevent non-specific labelling. The cells were incubated with a monoclonal antibody specific to Nrf2 (sc-722, Santa Cruz Biotechnology, Dallas, TX) overnight at $4^{\circ} \mathrm{C}$, then incubated with a secondary antibody, antirabbit IgG Fab2 Alexa Fluor 488 conjugate (Cell Signaling Technology, Inc., Beverly, MA) for $1 \mathrm{~h}$. Nuclei were stained with DAPI for $15 \mathrm{~min}$. Images were captured using a fluorescent microscopy BZ-X800 (Keyence Co., Osaka, Japan) at 400× magnification.

Extraction of protein and Western blot analysis. The nuclear protein extracts were prepared from $\mathrm{HaCaT}$ cells using NE-PER Nuclear and Cytoplasmic Extraction Kit (Thermo Scientific, Rockford, IL), according to the manufacturer's protocol. Additionally, total protein was extracted by ice-cold RIPA buffer (Nacalai Tesque) with protease inhibitor cocktail. The protein samples were subsequently prepared $4 \times$ Laemmli Sample Buffer (Bio-Rad Laboratories, Forester City, CA) and boiled at $100^{\circ} \mathrm{C}$ for $5 \mathrm{~min}$. Equal amounts of protein were separated by SDS-PAGE and then transferred onto polyvinylidene difluoride membranes for $35 \mathrm{~min}$ at $100 \mathrm{~V}$. After that, the membranes were blocked with Blocking One (Nacalai Tesque) for $40 \mathrm{~min}$ at room temperature. The membranes were incubated at $4^{\circ} \mathrm{C}$ overnight in each primary antibody. Primary antibodies specific to SIRT-1, HO-1, NF- $\kappa \mathrm{B}$, histone $\mathrm{H} 3$ and $\beta$-actin (Cell Signaling Technology) and Nrf2 were used. Membranes were washed by TBST three times and incubated with anti-rabbit or anti-mouse horseradish peroxidase-conjugated secondary antibody (Cell Signaling Technology). To detect the immunocomplex, an Immobilon HRP substrate (Bio-Rad Laboratories) was used. Immunoblots were scanned by densitometry and the intensity was quantified by using Image Lab software (Bio-Rad Laboratories). The relative levels of SIRT-1 and HO-1 were normalized to $\beta$-actin, and nuclear $\mathrm{Nrf} 2$ and $\mathrm{NF}-\kappa \mathrm{B}$ were standardized to histone $\mathrm{H} 3$.

Real-time PCR. HaCaT cells were sub-cultured and treated with 5-FU in the presence or absence of resveratrol and NAC (Sigma-Aldrich) for 24 and $48 \mathrm{~h}$. Total RNA was extracted using Trizol reagent (Invitrogen, Carlsbad, CA) according to the manufacturer's instructions. Total RNA was reverse transcribed using ReverTra Ace (Toyobo, Osaka, Japan). Real-time PCR was performed using SYBR Green (Bio-Rad Laboratories) and a real-time PCR system (Bio-Rad Laboratories) according to the following cycling parameters: initial denaturation at $95^{\circ} \mathrm{C}$ for $1 \mathrm{~min}, 40$ cycles of denaturation at $95^{\circ} \mathrm{C}$ for $20 \mathrm{~s}$, annealing at $60^{\circ} \mathrm{C}$ for $30 \mathrm{~s}$ and extension at $72^{\circ} \mathrm{C}$ for $25 \mathrm{~s}$. The primer sequences were showed in Table 1. The mRNA levels were calculated by determining the relative expression compared with GAPDH and the $\mathrm{Ct}$ of the control was normalized to 1 .

ELISA assay. HaCaT cells were seeded and preincubated for $24 \mathrm{~h}$. For the next $48 \mathrm{~h}$, the cells treated with 5-FU in the presence or absence of resveratrol. The culture supernatants were collected from both of treated and untreated cells, and centrifuged to remove cell debris. We used commercially available ELISA kits (Biolegend, San Diego, CA), according to the manufacturer's instructions. The concentrations of cytokines were calculated according to standard curves, and the obtained results are shown as the amount of IL- $1 \beta$, IL- 6 , and TNF- $\alpha$ $(\mathrm{pg} / \mathrm{ml})$.

Statistical analysis. For statistical analysis, differences between the groups were analyzed with one-way analysis of variance (ANOVA) and Tukey's post hoc test for multiple comparisons using JMP (ver. 12, SAS Institute, Cary, NC). A value of $p<0.05$ was regarded as statistically significant.

\section{Results}

Effects of resveratrol on 5-FU-induced cytotoxicity in $\mathrm{HaCaT}$ cells. The cytotoxicity of resveratrol and 5-FU $(5 \mu \mathrm{g} / \mathrm{ml})$ in $\mathrm{HaCaT}$ cells was evaluated after $24 \mathrm{~h}$ of treatment. We first examined the effects of various concentrations of resveratrol $(0.1-1,000 \mu \mathrm{M})$ on the viability of $\mathrm{HaCaT}$ cells in the presence and absence of 5-FU. Higher concentrations of resveratrol $(>100 \mu \mathrm{M})$ reduced $\mathrm{HaCaT}$ cell viability (Fig. 1A), whereas lower concentrations $(\leq 100 \mu \mathrm{M})$ had no influence on 
A

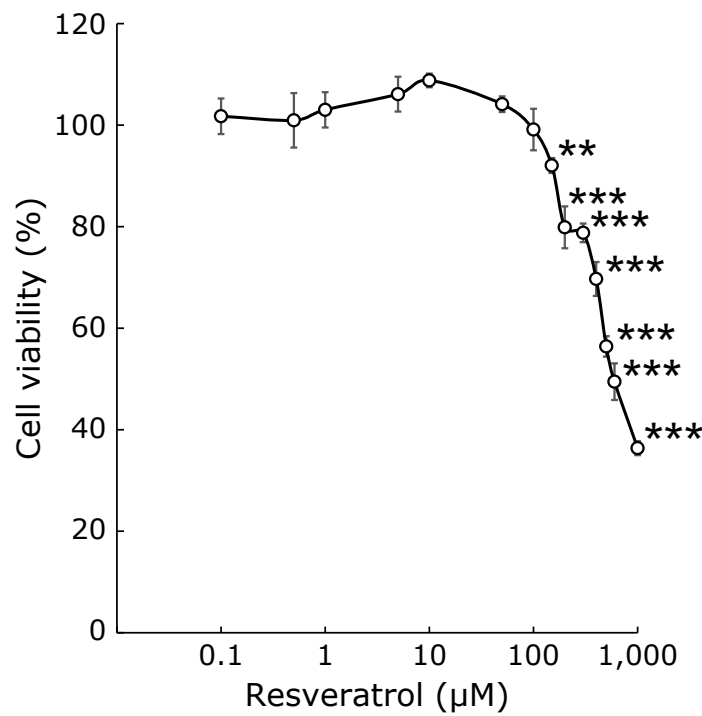

B

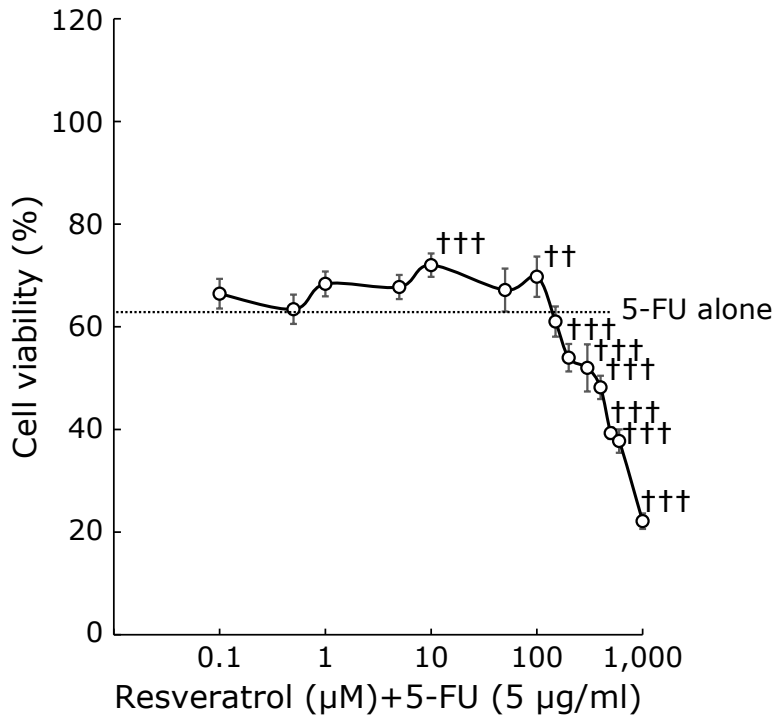

Fig. 1. Effects of resveratrol on 5-FU-induced cytotoxicity in HaCaT cells. HaCaT cells were treated with different concentrations of resveratrol alone $(A)$ or 5 -FU $(5 \mu \mathrm{g} / \mathrm{ml})$ and resveratrol (B) for $24 \mathrm{~h}$, and then cell viabilities were evaluated by WST-1 assay. The cell viabilities were expressed as $\%$ of control. Results are presented as the means \pm SD $(n=6)$. ${ }^{* *} p<0.01,{ }^{* * *} p<0.001$ significantly different from control. ${ }^{+t} p<0.01,{ }^{t+t} p<0.001$ significantly different from 5-FU alone.

A

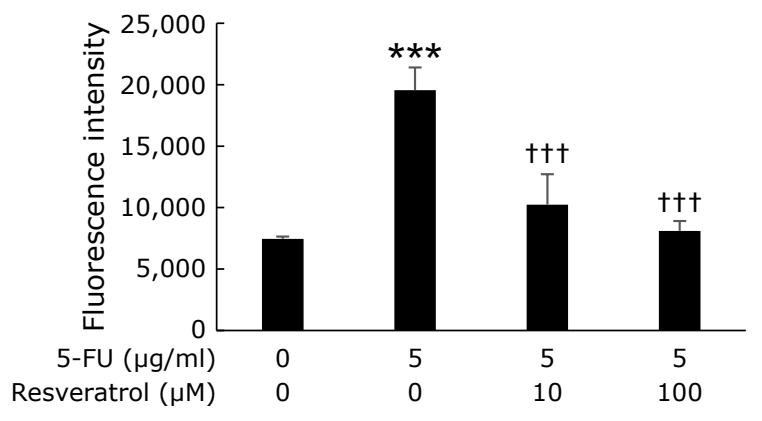

B

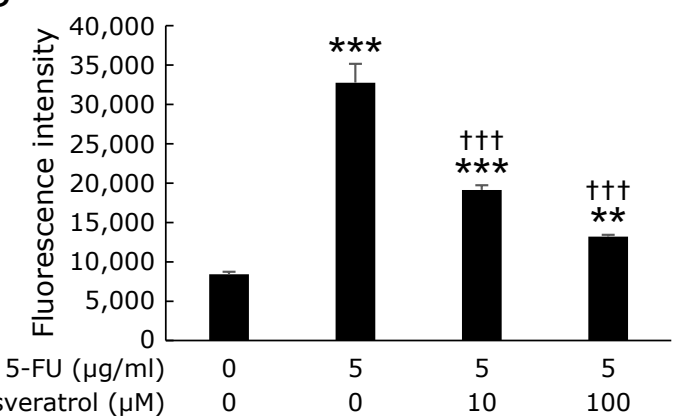

Fig. 2. Effects of resveratrol on the ROS production in HaCaT cells treated with 5-FU. HaCaT cells were incubated with 5-FU in the presence or absence of resveratrol for $24 \mathrm{~h}(\mathrm{~A})$ and $48 \mathrm{~h}$ (B). Results are presented as the means \pm SD of three independent experiments. ${ }^{* *} p<0.01, * * * p<0.001$ significantly different from control. ${ }^{+\dagger t} p<0.001$ significantly different from 5 -FU alone.

viability. In addition, 5-FU $(5 \mu \mathrm{g} / \mathrm{ml})$ treatment alone for $24 \mathrm{~h}$ significantly decreased cell viability relative to the control $(62.7$ $\pm 0.3 \%$ ). Although not all the lower concentrations of resveratrol had significantly increased the viability compared with 5-FU alone, there was a tendency for lower concentrations $(1-100 \mu \mathrm{M})$ to protect cytotoxicity induced by 5-FU (Fig. 1B).

Resveratrol suppresses 5-FU-induced ROS generation by activating the Nrf2 pathway in $\mathrm{HaCaT}$ cells. To investigate the anti-oxidant effects of resveratrol, we measured ROS production in $\mathrm{HaCaT}$ cells. 5-FU treatment for 24 or $48 \mathrm{~h}$ resulted in significantly increased ROS production (Fig. 2). Additionally, resveratrol significantly decreased ROS production induced by 5-FU treatment (Fig. 2).

We examined the effects of resveratrol on the Nrf2/anti-oxidant pathway. Nuclear expression of Nrf2 was slightly observed in 5-FU-treated $\mathrm{HaCaT}$ cells, but resveratrol increased nuclear $\mathrm{Nrf} 2$ expression as observed via immunofluorescence and western blot analyses using nuclear extracts (Fig. 3A and B). Thus, resveratrol accelerated Nrf2 nuclear translocation in HaCaT cells. Addition- ally, the expression of the anti-oxidant enzymes NQO-1 and HO-1 were analyzed by real-time PCR. 5-FU and resveratrol increased the expression of NQO-1 and HO-1 compared to their control levels (Fig. 3D and F). We also found that the protein expression of HO-1 was significantly increased by resveratrol treatment compared to the effects of 5-FU alone (Fig. 3C). Moreover, resveratrol alone also increased the expression of NQO-1 and HO-1 compared to control levels (Fig. 3E and G). These findings indicate that resveratrol activates the Nrf2 pathway and upregulates anti-oxidant defense genes.

Resveratrol increases the levels of SIRT-1 in HaCaT cells. Resveratrol is known as a SIRT-1 activator. Indeed, the mRNA expression of SIRT-1 was increased by resveratrol compared to control level in HaCaT cells (Fig. 4A). Additionally, we examined SIRT-1 expression after 5-FU treatment with or without resveratrol using real-time PCR and western blotting. 5-FU treatment increased the mRNA expression of SIRT-1 in HaCaT cells (Fig. 4B). Moreover, resveratrol synergistically enhanced both the protein and mRNA levels of SIRT-1 compared 
A

5 -FU $5 \mu \mathrm{g} / \mathrm{ml}$
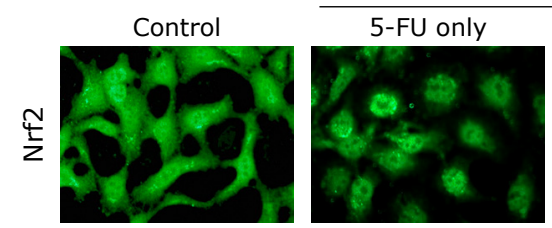

Resveratrol $10 \mu \mathrm{M}$ Resveratrol $100 \mu \mathrm{M}$
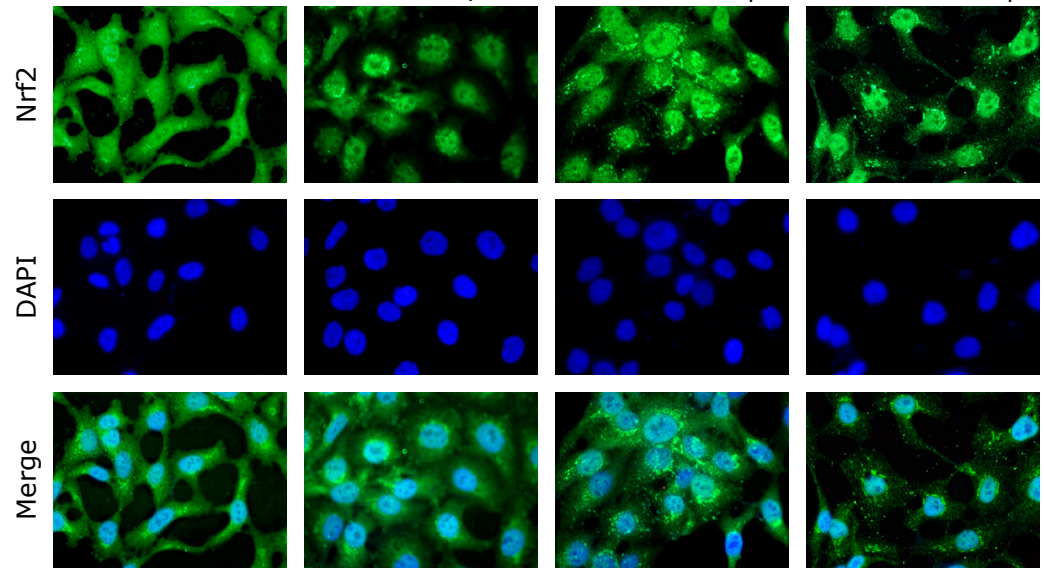

B

\section{C}
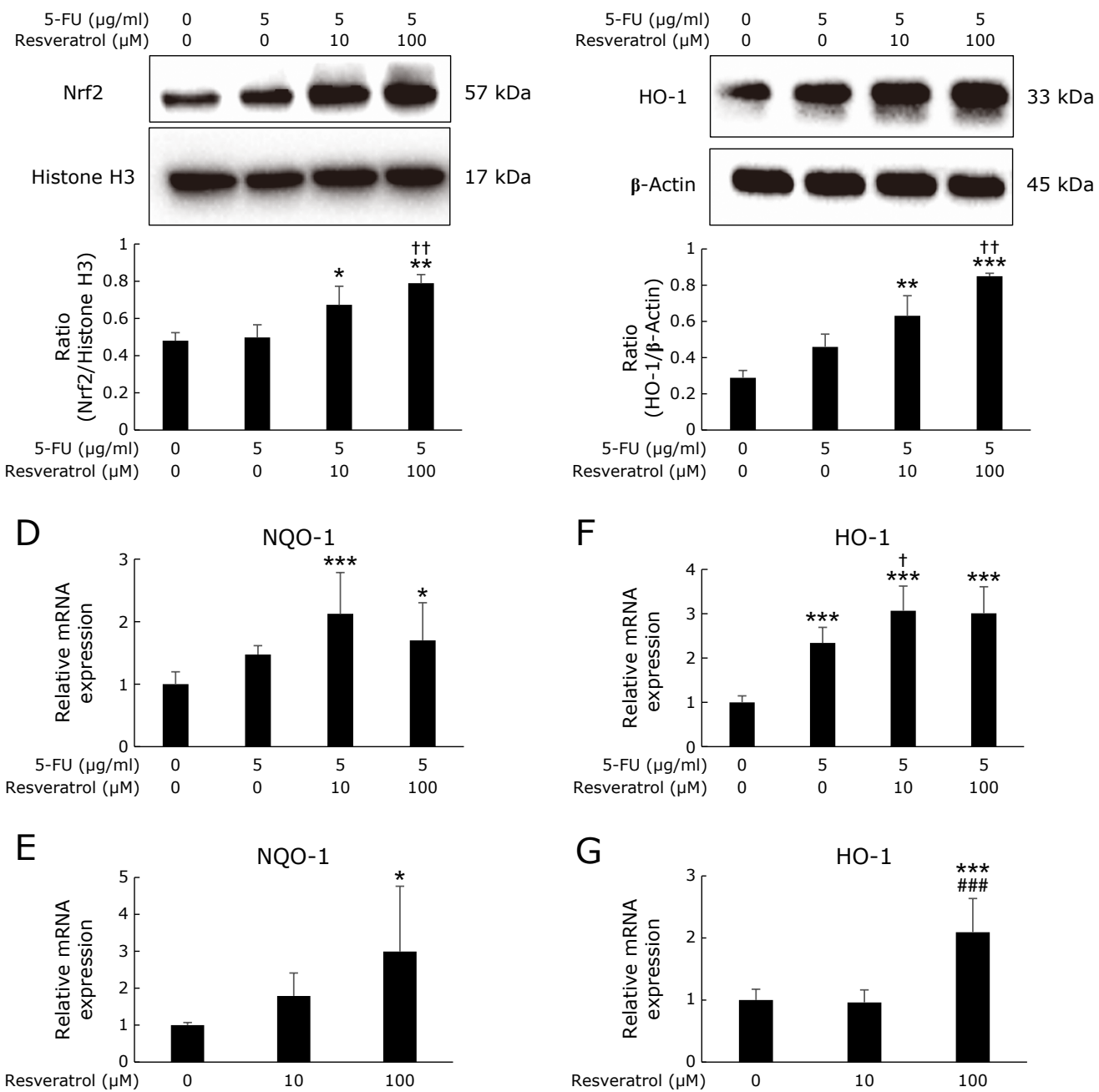

Fig. 3. Effects of resveratrol on the Nrf2 nuclear translocation, and expressions of HO-1 and NQO-1 in HaCaT cells treated with 5-FU. HaCaT cells incubated with 5-FU in the absence or presence of resveratrol for $24 \mathrm{~h}$. (A) Immunofluorescence staining of Nrf2 for each treatment. Nuclei were labeled with DAPI. 400x magnification. (B, C) Representative blots and quantitative analyses are shown for nuclear Nrf2, histone H3, HO-1, and $\beta$ actin. (D-G) Relative mRNA levels of NQO-1 and HO-1 were determined by real-time PCR. Bars represent mRNA expression normalized to GAPDH and relative to the control. Results are presented as the means \pm SD $(n=6)$. ${ }^{*} p<0.05, * * p<0.01, * * * p<0.001$ significantly different from control.

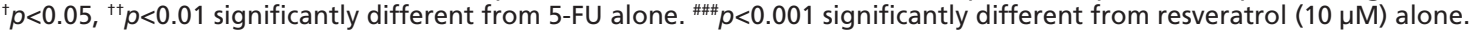



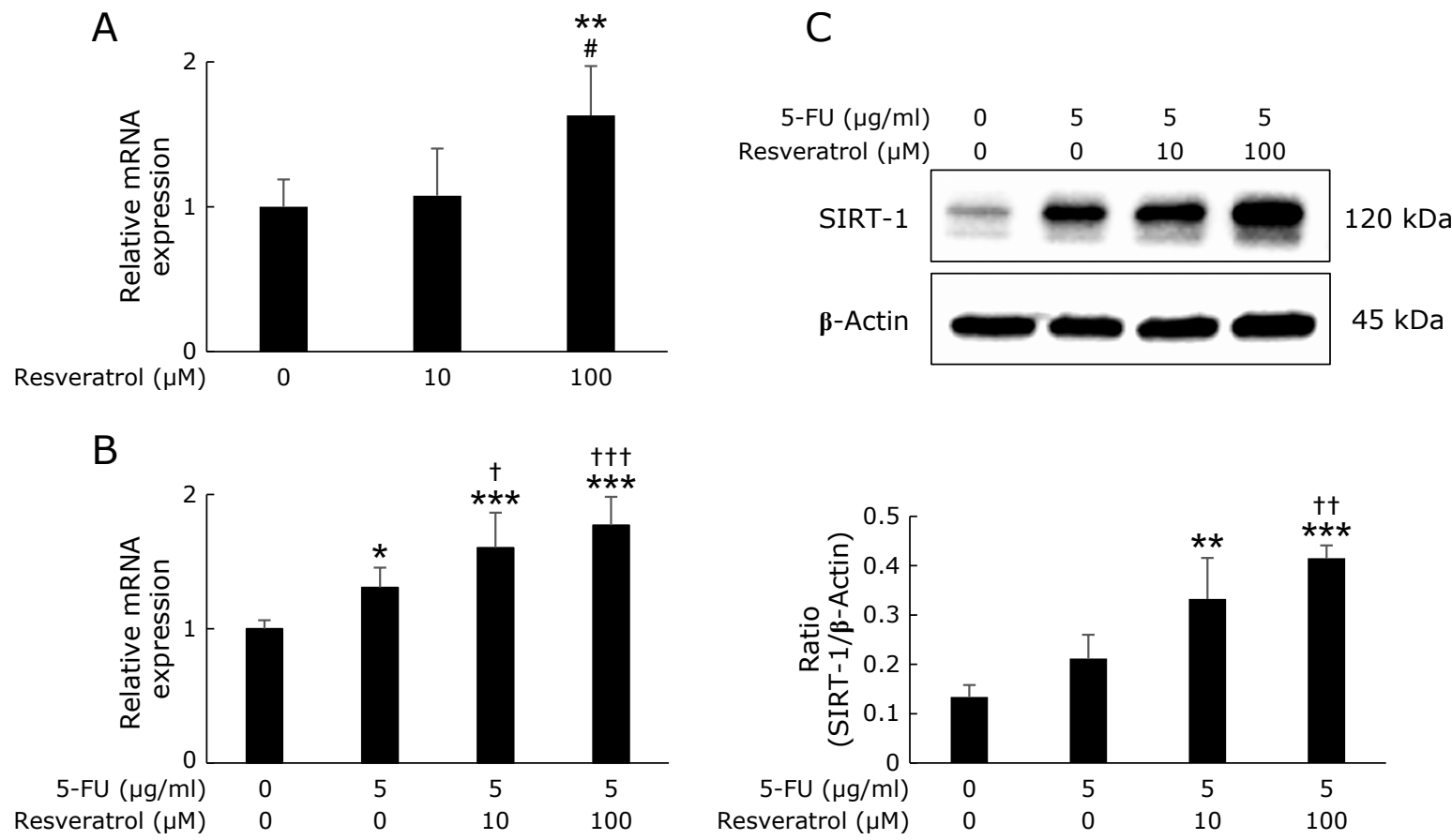

Fig. 4. Effects of resveratrol on the levels of SIRT-1 in HaCaT cells treated with 5-FU. HaCaT cells incubated with 5-FU in the absence or presence of resveratrol for $24 \mathrm{~h}$. (A, B) Relative mRNA levels of SIRT-1 were determined by real-time PCR. Bars represent mRNA expression normalized to GAPDH and relative to the control. (C) Representative blots are shown for SIRT- 1 and $\beta$-actin. Quantitative analyses are shown for SIRT- $1 / \beta$-actin. ${ }^{*} p<0.05,{ }^{*} p<0.01, * * * p<0.001$ significantly different from control. ${ }^{\dagger} p<0.05,{ }^{+\dagger} p<0.01,{ }^{+t} p<0.001$ significantly different from 5 -FU alone. ${ }^{\#} p<0.05$ significantly different from resveratrol $(10 \mu \mathrm{M})$ alone.

to the effects of 5-FU alone (Fig. 4B and C).

Resveratrol inhibits 5-FU-induced NF-kB p65 nuclear translocation in HaCaT cells. The effect of resveratrol on the 5 -FU-induced nuclear translocation of the NF- $\mathrm{BB}$ p65 subunit was examined (Fig. 5A). NF- $\kappa \mathrm{B}$ p65 expression in the nucleus was significantly increased by 5-FU treatment, and resveratrol reduced the 5-FU-induced nuclear expression of p65. It is thus suggested that resveratrol inhibits the NF- $\kappa \mathrm{B}$ pathway, which is activated by 5-FU. We also examined the effect of resveratrol alone on NF- $\kappa \mathrm{B}$ expression. Resveratrol treatment had no significant influence on its expression (Fig. 5B).

Resveratrol suppresses 5-FU-induced inflammatory cytokine expression in $\mathrm{HaCaT}$ cells. The mRNA and protein levels of inflammatory cytokines in $\mathrm{HaCaT}$ cells were measured by real-time PCR (Fig. 6A-H) and ELISA (Fig. 6I and J), respectively. Significantly higher expression of IL-1 $\beta$, IL- 6 , and TNF- $\alpha$ was observed after exposure to 5-FU at different time points (24 and $48 \mathrm{~h}$; Fig. $6 \mathrm{~A}-\mathrm{C}$ and $6 \mathrm{E}-\mathrm{G}$, respectively). Resveratrol significantly decreased the expression of IL-1 $\beta$, IL-6, and TNF- $\alpha$ induced by $48 \mathrm{~h}$ of 5 -FU treatment (Fig. $6 \mathrm{E}-\mathrm{G}$ ). Further, the protein levels of IL-1 $\beta$ and IL-6, which were increased by 5-FU treatment, were also reduced after $48 \mathrm{~h}$ of resveratrol treatment (Fig. 6I-J). TNF- $\alpha$ protein levels were under the detection limit of ELISA in all conditions. We also investigated the effect of resveratrol on IL-10 expression, finding that the treatment had no significant influence on its expression (Fig. 6D, H, and K).

NAC suppresses 5 -FU-induced ROS production but not pro-inflammatory cytokine expression. We examined whether NAC could decrease the expression of pro-inflammatory cytokines by suppressing the overproduction of ROS. As expected, NAC significantly decreased ROS production induced by $5-\mathrm{FU}$ treatment (Fig. 7A). However, NAC did not reduce the expression of inflammatory cytokines induced by 5-FU treatment at 24 (Fig. 7B-D) and $48 \mathrm{~h}$ (Fig. 7E-G). This finding suggests that the expression of inflammatory cytokines in HaCaT cells following 5-FU treatment is not a downstream event of ROS production and is independent of oxidative stress.

\section{Discussion}

In this study, we explored the utility of resveratrol for treating chemotherapy-induced oral mucositis using an in vitro cell culture model. To the best of our knowledge, this was the first study to demonstrate the protective effects of resveratrol on oxidative stress and inflammation induced by chemotherapy in normal human keratinocytes. Additionally, our findings suggest that resveratrol exerts substantial anti-oxidant and antiinflammatory effects by activating Nrf2 signaling and inhibiting $\mathrm{NF}-\kappa \mathrm{B}$ signaling, respectively.

5-FU has excellent effects against a variety of cancers including breast, head and neck, stomach, and skin cancers. ${ }^{(2)}$ 5-FU blocks DNA replication and induces the overproduction of ROS and upregulation of inflammatory responses, thereby directly inducing toxicity in the oral mucosa. ${ }^{(17-19)} 5$-FU commonly causes oral mucositis in patients with cancer. ${ }^{(1)}$ The pathogenesis of oral mucositis is divided into five biological steps: (i) initiation, (ii) primary damage response, (iii) signal amplification, (iv) ulceration, and (v) healing. ${ }^{(4)}$ During the initial stage of oral mucositis, anti-cancer drugs induce the overproduction of ROS, which play a primary role in the activation of a variety of transcription factors, such as NF- $\kappa \mathrm{B} .{ }^{(20)}$ Activation of $\mathrm{NF}-\kappa \mathrm{B}$ subsequently leads to the production of the inflammatory cytokines IL-1 $\beta$, IL-6, and TNF- $\alpha{ }^{(19)}$ The inflammatory cytokines interact synergistically with $\mathrm{NF}-\kappa \mathrm{B}$, thereby amplifying the initial signals toward cell damage and tissue destruction, which occur in the primary damage response of oral mucositis. ${ }^{(20,21)}$ We revealed that the nuclear translocation of NF- $\kappa$ B p65 (Fig. 5) and production of pro-inflammatory cytokines (Fig. 6) were significantly induced by 5 -FU treatment in $\mathrm{HaCaT}$ cells, suggesting that the nuclear translocation of NF- $\kappa \mathrm{B}$ p65 

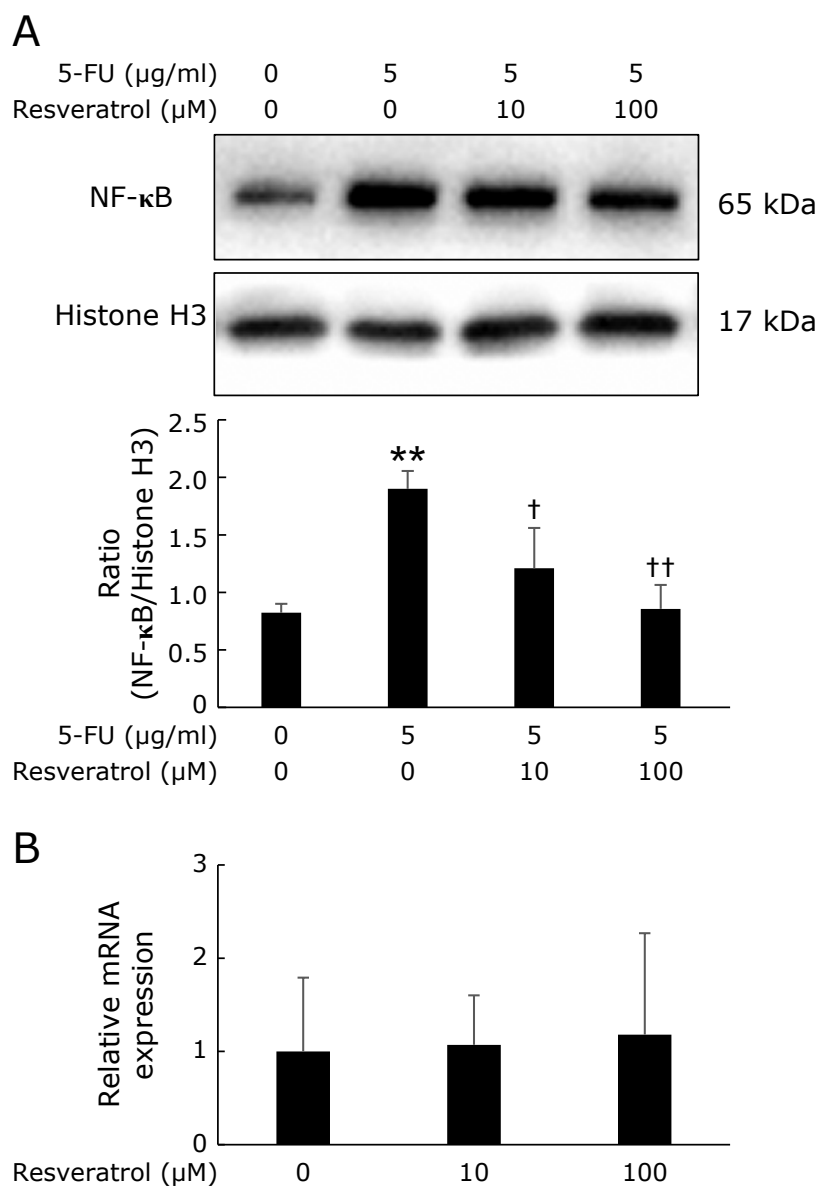

Fig. 5. Effects of resveratrol on nuclear translocation of NF-kB in $\mathrm{HaCaT}$ cells treated with 5-FU. (A) HaCaT cells were pre-treated with resveratrol for $3 \mathrm{~h}$ and then added $5 \mu \mathrm{g} / \mathrm{ml}$ of 5 -FU for $6 \mathrm{~h}$. Representative Western blots and quantitative analysis were shown. (B) Relative mRNA levels of NF- $\kappa B$ were determined by real-time PCR. Bars represent mRNA expression normalized to GAPDH and relative to the control. ${ }^{* *} p<0.01$ significantly different from control. ${ }^{\dagger} p<0.05,{ }^{+\dagger} p<0.01$ significantly different from 5-FU alone.

leads to the upregulation of pro-inflammatory cytokines.

5 -FU treatment increased the levels of ROS in HaCaT cells (Fig. 2). There is a balance between the production and elimination of ROS in the healthy condition. However, chronic inflammation leads to sustained ROS overproduction. Therefore, it is believed that the uptake of exogenous anti-oxidants is beneficial for eliminating ROS and controlling oxidative stress in the body. ${ }^{(22,23)}$ Previous researches have illustrated that resveratrol is the efficient hydroxyl radical and superoxide radical scavenger, which is substantial but less effective than known radical scavengers such as glutathione and ascorbic acid. ${ }^{(22,24)}$ In addition, resveratrol has been reported to increase the levels of antioxidant genes, i.e., Nrf2, HO-1, and SIRT-1, in normal cells. ${ }^{(25,26)}$ It has been also reported that resveratrol can regulate the expression and activity of anti-oxidant enzymes via the activation of the transcription factor Nrf2.(27) Our findings demonstrated that resveratrol could reduce the overproduction of ROS induced by 5-FU in HaCaT cells (Fig. 2). Moreover, resveratrol can promote the activity of SIRT-1, which regulates the activity of multiple downstream targets including the NF- $\mathrm{BB}$ pathway. ${ }^{(28,29)}$ Consistent with these previous studies, resveratrol could controll oxidative stress with activating of Nrf2/anti-oxidant and SIRT-1 defense pathways as well as ROS scavenging.

$\mathrm{Nrf} 2$ is considered a main regulator of anti-oxidant defenses in mammals. ${ }^{(30)}$ In response to oxidative stress, Nrf2 is activated, and it translocates to the nucleus, in which it binds to AREs and upregulates the expression of phase 2 enzymes, such as HO-1 and NQO- 1 , thereby maintaining the redox balance of cells. ${ }^{(31,32)}$ We found that the nuclear translocation of $\mathrm{Nrf} 2$ was more strongly increased in $\mathrm{HaCaT}$ cells treated with both 5-FU and resveratrol than in cells treated with 5-FU alone (Fig. 3). In addition, the upregulation of HO-1 and NQO-1 expression was significantly induced by resveratrol (Fig. 3). As 5-FU-induced ROS overproduction was significantly suppressed by resveratrol (Fig. 2), the compound might regulate oxidative stress by activating the Nrf2/anti-oxidant pathway in normal cells.

SIRT-1 is a nicotinamide adenine dinucleotide-dependent nuclear histone deacetylase that participates in the regulation of several processes, such as inflammation, histone deacetylation, and apoptosis. ${ }^{(33-35)}$ SIRT-1 reduces the levels of oxidative stress and the severity of inflammation. ${ }^{(36,37)}$ Moreover, SIRT-1 promotes the nuclear accumulation, DNA binding, and transcriptional activities of Nrf2 by upregulating its downstream genes HO-1 and NQO-1. ${ }^{(38)}$ In addition, the inhibition of Nrf2 ubiquitination by SIRT-1, which increases the stability and activity of $\mathrm{Nrf2}$, suppresses apoptosis and resistance to oxidative stress. ${ }^{(39)}$ In this study, we illustrated that resveratrol enhanced both SIRT-1 and nuclear Nrf2 levels in HaCaT cells (Fig. 3 and 4), suggesting that SIRT-1 promotes the stability and activity of Nrf2 under treatment with resveratrol. SIRT-1 also inhibits the transactivation of NF- $\kappa \mathrm{B} .{ }^{(35)}$ In addition, HO-1 has a central role in NF- $\mathrm{BB}$ inhibition in the cytoplasm. ${ }^{(40)}$ Therefore, we speculate that resveratrol can suppress the inflammatory responses activated by $\mathrm{NF}-\kappa \mathrm{B}$ through upregulating SIRT-1 and HO-1.

$\mathrm{NF}-\kappa \mathrm{B}$ activation is a cellular injury response that leads to inflammation via the production of pro-inflammatory cytokines, such as IL-1 $\beta$, IL- 6 , and TNF- $\alpha .{ }^{(41)}$ In an inactive state, NF- $\kappa$ B exists in the cytoplasm as a heterodimer of p65 and p50 subunits, but once activated by the phosphorylation and degradation of inhibitor of kappa B, it translocates to the nucleus and activates the transcription of various pro-inflammatory genes. ${ }^{(42)}$ Resveratrol does not affect the DNA-binding activity of NF- $\kappa B$, but it inhibits the nuclear translocation of $\mathrm{p} 65 .^{(43)}$ In the present study, differing from resveratrol, NAC significantly decreased the overproduction of ROS induced by 5-FU, and it did not affect the expression of inflammatory cytokines (Fig. 7). This suggests that 5-FU directly induces the upregulation of inflammatory cytokines rather than through the overproduction of ROS. Importantly, resveratrol might protect cells against the cytotoxicity of 5-FU via two independent pathways, i.e., anti-oxidant and antiinflammatory signaling. In the latter, suppression of the 5-FUinduced nuclear translocation of NF- $\mathrm{kB}$ p65 is likely responsible for the downregulation of inflammatory cytokines.

In conclusion, resveratrol displayed multiple beneficial effects in an in vitro oral mucositis model induced by 5-FU. Resveratrol decreased 5-FU-induced ROS production by activating the Nrf2 and SIRT-1 pathways in normal human keratinocytes. Moreover, resveratrol suppressed inflammatory responses by reducing the production of inflammatory cytokines via the suppression of $\mathrm{NF}-\kappa \mathrm{B}$ signaling. Overall, our results support the hypothesis that resveratrol is a safe and beneficial candidate for preventing oral mucositis associated with cancer chemotherapy. However, further studies are required before the practical use of resveratrol supplements to prevent oral mucositis.

\section{Author Contributions}

SC contributed to perform all experiments and drafted the manuscript. NT contributed to design the study, perform experiments, and revise manuscript. YK contributed to perform immunofluorescence staining, and revise manuscript. TT and KM contributed to perform Western blot analysis. NI and HI 

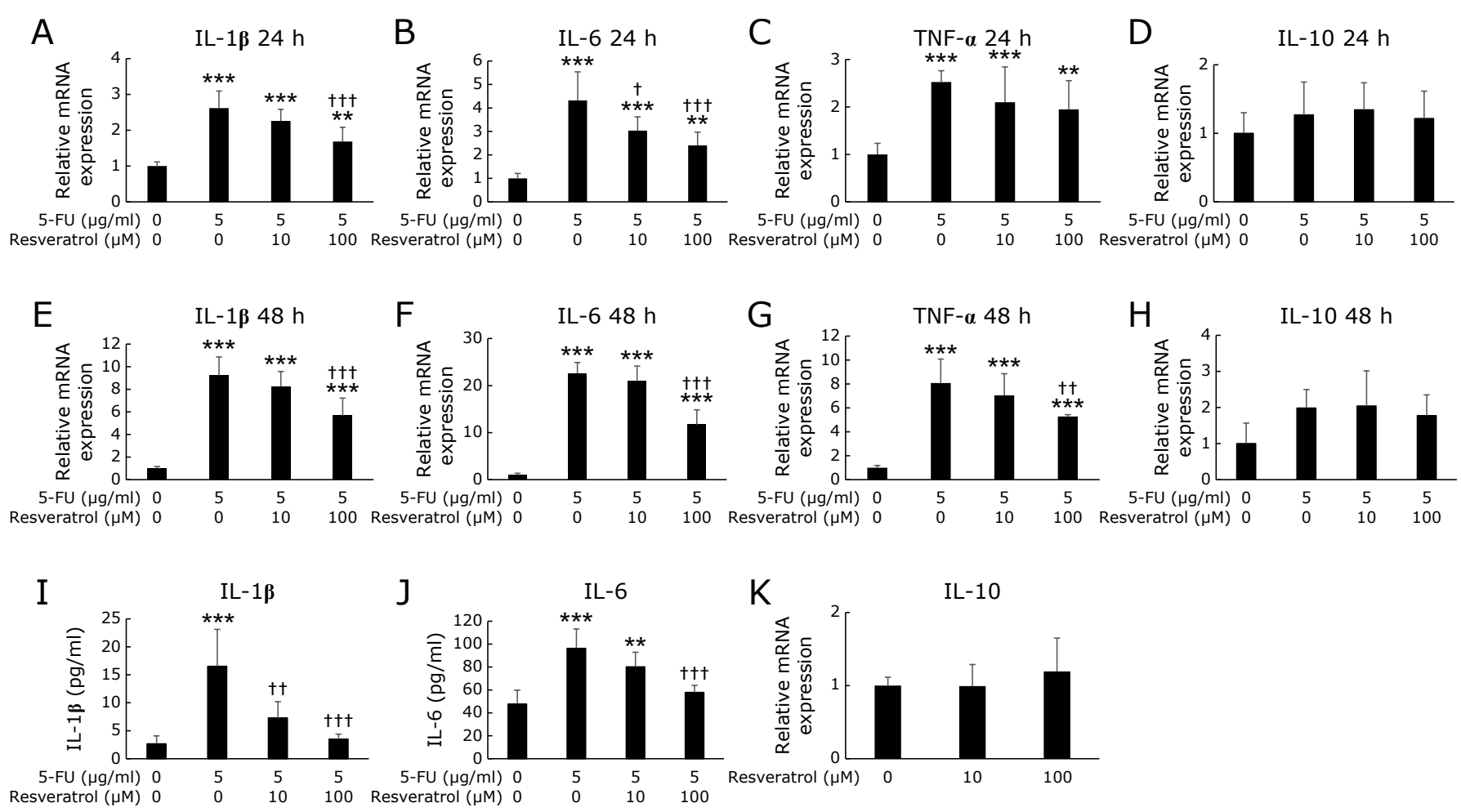

Fig. 6. Effects of resveratrol on 5-FU induced-inflammatory cytokines in HaCaT cells treated with 5-FU. HaCaT cells were treated with 5-FU in the presence or absence of resveratrol for $24 \mathrm{~h}(\mathrm{~A}-\mathrm{D}, \mathrm{K})$ and $48 \mathrm{~h}(\mathrm{E}-\mathrm{H})$. IL-1 $\beta$, IL-6, TNF- $\alpha$, and IL-10 levels were determined by real-time PCR. Bars represent mRNA expression normalized to GAPDH and relative to control. (I) IL-1 $\beta$ and (J) IL- 6 amounts of culture supernatants of HaCaT cells at 48 $\mathrm{h}$ were determined by ELISA. Data are expressed as means \pm SD $(n=6) .{ }^{* *} p<0.01,{ }^{* * *} p<0.001$ significantly different from control. ${ }^{+} p<0.05,{ }^{+\dagger} p<0.01$, ${ }^{t+t} p<0.001$ significantly different from 5 -FU alone.
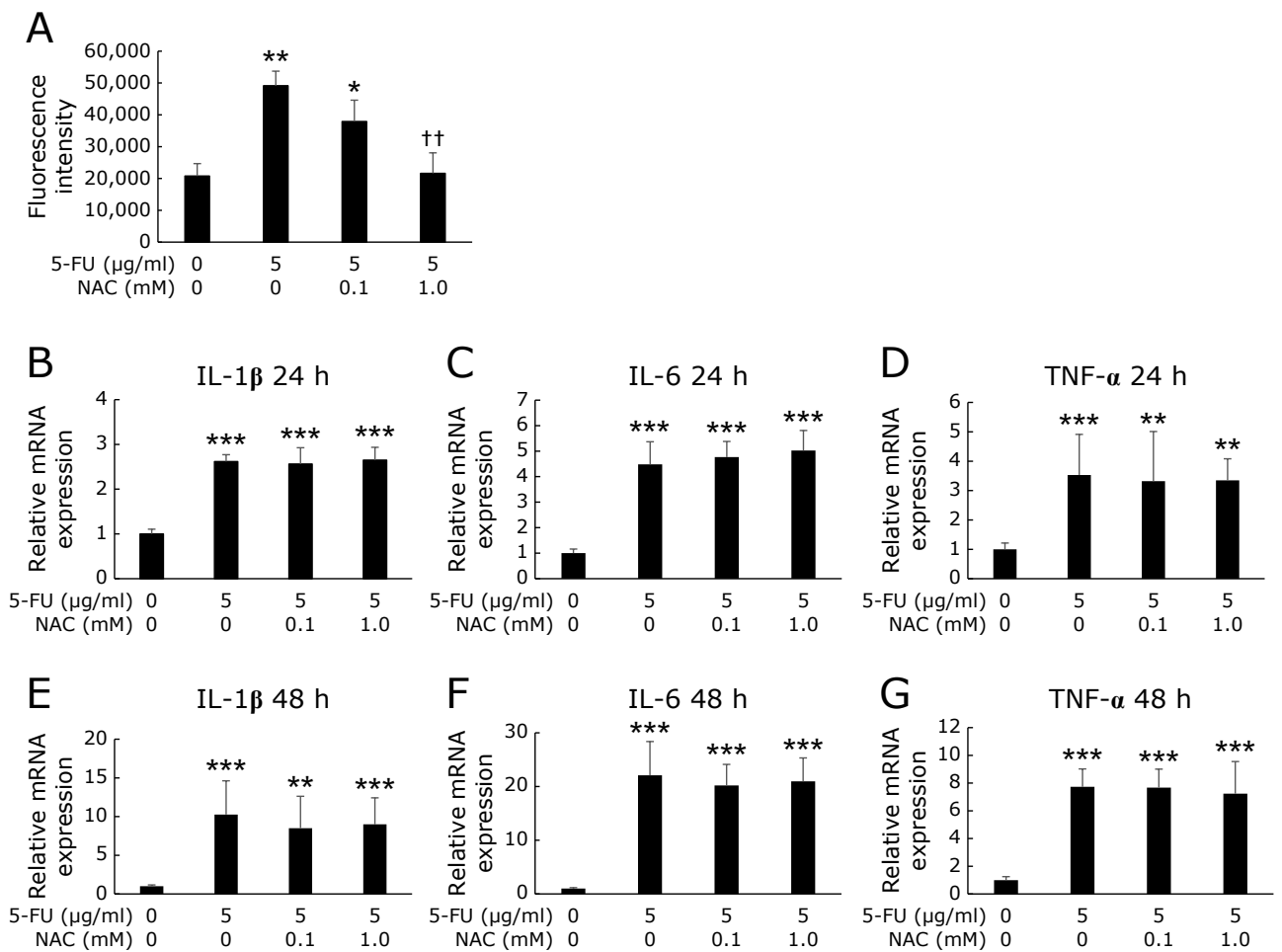

Fig. 7. Effects of NAC on the levels of production of ROS and pro-inflammatory cytokines in 5-FU treated HaCaT cells. HaCaT cells was incubated with 5 -FU in the presence or absence of NAC for $24 \mathrm{~h}(A-D)$ and $48 \mathrm{~h}(\mathrm{E}-\mathrm{G})$. (A) ROS assay. Results are presented as the means \pm SD of three independent experiments. (B-G) IL-1 $\beta$, IL-6, and TNF- $\alpha$ levels were determined by real-time PCR. Bars represent mRNA expression normalized to GAPDH and relative to control. Data are expressed as means \pm SD $(n=6) .{ }^{*} p<0.05,{ }^{* *} p<0.01,{ }^{* * *} p<0.001$, significantly different from control. ${ }^{+\dagger} p<0.01$ significantly different from 5-FU alone. 
contributed to revise manuscript. All the authors read and approved the final manuscript.

\section{Acknowledgments}

This work was supported by Grants-in-Aid for Scientific Research (JSPS KAKENHI Grant Numbers JP18K09911 and JP21K10255) from the Ministry of Education, Culture, Sports, Science and Technology of Japan.

\section{Abbreviations}

$\begin{array}{ll}\text { ARE } & \text { antioxidant response element } \\ \text { 5-FU } & \text { 5-fluorouracil } \\ \text { HaCaT cell } & \text { immortalized human keratinocyte cell line } \\ \text { HO-1 } & \text { heme oxygenase 1 }\end{array}$

\section{References}

1 Curra M, Soares Junior LAV, Martins MD, Santos PSDS. Chemotherapy protocols and incidence of oral mucositis. An integrative review. Einstein (São Paulo) 2018; 16: eRW4007.

2 Rashid S, Ali N, Nafees S, Ahmad ST, Hasan SK, Sultana S. Abrogation of 5 flourouracil induced renal toxicity by bee propolis via targeting oxidative stress and inflammation in Wistar rats. J Pharm Pharmacol 2013; 7: 189-194.

3 Epstein JB, Robertson M, Emerton S, Phillips N, Stevenson-Moore P. Quality of life and oral function in patients treated with radiation therapy for head and neck cancer. Head Neck 2001; 23: 389-398.

4 Sonis ST. A biological approach to mucositis. J Support Oncol 2004; 2: 21 36.

5 Henke M, Alfonsi M, Foa P, et al. Palifermin decreases severe oral mucositis of patients undergoing postoperative radiochemotherapy for head and neck cancer: a randomized, placebo-controlled trial. J Clin Oncol 2011; 29: 2815 2820 .

6 Chang JW, Park KH, Hwang HS, Shin YS, Oh YT, Kim CH. Protective effects of Korean red ginseng against radiation-induced apoptosis in human HaCaT keratinocytes. J Radiat Res 2014; 55: 245-256.

7 Sharma P, Jha AB, Dubey RS, Pessarakli M. Reactive oxygen species, oxidative damage, and antioxidative defense mechanism in plants under stressful conditions. J Bot 2012; 2012: 217037.

8 Rogers $\mathrm{H}$, Munné-Bosch S. Production and scavenging of reactive oxygen species and redox signaling during leaf and flower senescence: similar but different. Plant Physiol 2016; 171: 1560-1568.

9 Loboda A, Damulewicz M, Pyza E, Jozkowicz A, Dulak J. Role of Nrf2/ HO-1 system in development, oxidative stress response and diseases: an evolutionarily conserved mechanism. Cell Mol Life Sci 2016; 73: 3221-3247.

10 Yang HL, Lin SW, Lee CC, et al. Induction of Nrf2-mediated genes by Antrodia salmonea inhibits ROS generation and inflammatory effects in lipopolysaccharide-stimulated RAW264.7 macrophages. Food Funct 2015; 6: $230-241$.

11 Fu Q, Cui Q, Yang Y, et al. Effect of resveratrol dry suspension on immune function of piglets. Evid Based Complement Alternat Med 2018; 2018: 5952707.

12 Orihuela-Campos RC, Tamaki N, Mukai R, et al. Biological impacts of resveratrol, quercetin, and $N$-acetylcysteine on oxidative stress in human gingival fibroblasts. J Clin Biochem Nutr 2015; 56: 220-227.

13 Xiao Q, Zhu W, Feng W, et al. A review of resveratrol as a potent chemoprotective and synergistic agent in cancer chemotherapy. Front Pharmacol 2019; 9: 1534 .

14 Dos Santos Filho EX, da Silva ACG, de Ávila RI, et al. Chemopreventive effects of FITOPROT against 5-fluorouracil-induced toxicity in $\mathrm{HaCaT}$ cells Life Sci 2018; 193: 300-308.

15 Partridge M, Chantry D, Turner M, Feldmann M. Production of interleukin-1 and interleukin- 6 by human keratinocytes and squamous cell carcinoma cell lines. J Invest Dermatol 1991; 96: 771-776.

16 Konur A, Schulz U, Eissner G, Andreesen R, Holler E. Interferon (IFN)gamma is a main mediator of keratinocyte $(\mathrm{HaCaT})$ apoptosis and contributes to autocrine IFN-gamma and tumour necrosis factor-alpha production. $\mathrm{Br} J$
IL-1 $\beta$
IL-6
IL-10
NAC
NAD
$\mathrm{NF}-\kappa \mathrm{B}$
NQO-1
$\mathrm{Nrf} 2$
ROS
SIRT-1
TNF- $\alpha$
interleukin (IL)- $1 \beta$
interleukin (IL)-6
interleukin (IL)-10
$\mathrm{N}$-acetylcysteine
nicotinamide adenine dinucleotide
nuclear factor kappa B
$\mathrm{NAD}(\mathrm{P}) \mathrm{H}$ and quinone oxidoreductase 1 nuclear factor erythroid 2-related factor 2
reactive oxygen species
silent information regulator transcript-1
tumor necrosis factor- $\alpha$

\section{Conflicts of Interest}

No potential conflicts of interest were disclosed.

Dermatol 2005; 152: 1134-1142.

17 Kim DR, Kim J, Oh JY, Kim HY, Kim YJ, Chang MS. Protective effect of Salvia miltiorrhiza Bunge on 5-fluorouracil-induced oral mucositis. Int $\mathrm{J} \mathrm{Mol}$ Med 2017; 40: 39-46.

18 Kang MA, So EY, Simons AL, Spitz DR, Ouchi T. DNA damage induces reactive oxygen species generation through the H2AX-Nox1/Rac1 pathway. Cell Death Dis 2012; 3: e249.

19 Barbosa MM, de Araújo AA, de Araújo Júnior RF, et al. Telmisartan modulates the oral mucositis induced by 5-Fluorouracil in hamsters. Front Physiol 2018; 9: 1204.

20 Sonis ST. Pathobiology of oral mucositis: novel insights and opportunities. $J$ Support Oncol 2007; 5 (9 Suppl 4): 3-11.

21 Harris DJ. Cancer treatment-induced mucositis pain: strategies for assessment and management. Ther Clin Risk Manag 2006; 2: 251-258.

22 Leonard SS, Xia C, Jiang BH, et al. Resveratrol scavenges reactive oxygen species and effects radical-induced cellular responses. Biochem Biophys Res Commun 2003; 309: 1017-1026.

23 Kurutas EB. The importance of antioxidants which play the role in cellular response against oxidative/nitrosative stress: current state. Nutr $J$ 2016; 15: 71.

24 Singh N, Agrawal M, Doré S. Neuroprotective properties and mechanisms of resveratrol in in vitro and in vivo experimental cerebral stroke models. ACS Chem Neurosci 2013; 4: 1151-1162.

25 Shen $\mathrm{C}$, Cheng W, Yu P, et al. Resveratrol pretreatment attenuates injury and promotes proliferation of neural stem cells following oxygen-glucose deprivation/reoxygenation by upregulating the expression of Nrf2, HO-1 and NQO-1 in vitro. Mol Med Rep 2016; 14: 3646-3654.

26 Dong W, Wang X, Bi S, et al. Inhibitory effects of resveratrol on foam cell formation are mediated through monocyte chemotactic protein-1 and lipid metabolism-related proteins. Int J Mol Med 2014; 33: 1161-1168.

27 Dong WW, Liu YJ, Lv Z, et al. Lung endothelial barrier protection by resveratrol involves inhibition of HMGB1 release and HMGB1-induced mitochondrial oxidative damage via an Nrf2-dependent mechanism. Free Radic Biol Med 2015; 88 (Pt B): 404-416.

28 Chao SC, Chen YJ, Huang $\mathrm{KH}$, et al. Induction of sirtuin-1 signaling by resveratrol induces human chondrosarcoma cell apoptosis and exhibits antitumor activity. Sci Rep 2017; 7: 3180.

29 Ren Z, He H, Zuo Z, Xu Z, Wei Z, Deng J. The role of different SIRT1mediated signaling pathways in toxic injury. Cell Mol Biol Lett 2019; 24: 36.

30 Mukaigasa K, Nguyen LT, Li L, Nakajima H, Yamamoto M, Kobayashi M. Genetic evidence of an evolutionarily conserved role for Nrf2 in the protection against oxidative stress. Mol Cell Biol 2012; 32: 4455-4461.

31 Nguyen T, Nioi P, Pickett CB. The Nrf2-antioxidant response element signaling pathway and its activation by oxidative stress. J Biol Chem 2009; 284: 13291-13295.

32 Li L, Dong H, Song E, Xu X, Liu L, Song Y. Nrf2/ARE pathway activation, HO-1 and NQO-1 induction by polychlorinated biphenyl quinone is associated with reactive oxygen species and PI3K/AKT signaling. Chem Biol Interact 2014; 209: 56-67. 
33 Wang S, Wang J, Zhao A, Li J. SIRT1 activation inhibits hyperglycemiainduced apoptosis by reducing oxidative stress and mitochondrial dysfunction in human endothelial cells. Mol Med Rep 2017; 16: 3331-3338.

34 Han MK, Song EK, Guo Y, Ou X, Mantel C, Broxmeyer HE. SIRT1 regulates apoptosis and nanog expression in mouse embryonic stem cells by controlling p53 subcellular localization. Cell Stem Cell 2008; 2: 241-251.

35 Yeung F, Hoberg JE, Ramsey CS, et al. Modulation of NF- $\kappa B-d e p e n d e n t$ transcription and cell survival by the SIRT1 deacetylase. EMBO J 2004; 23: 2369-2380.

36 Rada P, Pardo V, Mobasher MA, et al. SIRT1 controls acetaminophen hepatotoxicity by modulating inflammation and oxidative stress. Antioxid Redox Signal 2018; 28: 1187-1208.

37 Chan SH, Hung CH, Shih JY, et al. SIRT1 inhibition causes oxidative stress and inflammation in patients with coronary artery disease. Redox Biol 2017; 13: 301-309.

38 Huang KP, Huang J, Xie X, et al. Sirt1 resists advanced glycation end products-induced expressions of fibronectin and TGF- $\beta 1$ by activating the Nrf2/ARE pathway in glomerular mesangial cells. Free Radic Biol Med 2013; 65: $528-540$.
39 Ding YW, Zhao GJ, Li XL, et al. SIRT1 exerts protective effects against paraquat-induced injury in mouse type II alveolar epithelial cells by deacetylating NRF2 in vitro. Int J Mol Med 2016; 37: 1049-1058.

40 Wardyn JD, Ponsford AH, Sanderson CM. Dissecting molecular cross-talk between Nrf2 and NF-kB response pathways. Biochem Soc Trans 2015; 43: 621-626.

41 Zhang Z, Liang Z, Li $\mathrm{H}$, et al. Perfluorocarbon reduces cell damage from blast injury by inhibiting signal paths of NF- $\mathrm{B}$, MAPK and Bcl-2/Bax signaling pathway in A549 cells. PLoS One 2017; 12: e0173884.

42 Albensi BC. What is nuclear factor kappa B $(\mathrm{NF}-\mathrm{kB})$ doing in and to the mitochondrion? Front Cell Dev Biol 2019; 7: 154.

43 Pervaiz S. Resveratrol: from grapevines to mammalian biology. FASEB $J$ 2003; 17: 1975-1985.

This is an open access article distributed under the terms of the Creative Commons Attribution-NonCommercial-NoDerivatives License (http://creativecommons.org/licenses/by-nc-nd/4.0/). 